\title{
HOW FLEXIBLE IS YOUR TRAUMA SPLINT?
}

\section{By Shola} Todd

1 splint is required when teeth are mobile or need to be repositioned following a traumatic injury. Dental trauma can result in considerable cost to the health service, patients and parents because long term dental care is often required. Furthermore, severe dental trauma almost always involves the upper anterior teeth with considerable effect on dental and facial aesthetics thus impacting quality of life.

The aims of splinting are:

- To stabilise the tooth/teeth that have been loosened, repositioned, replanted or fractured and maintain its/their position throughout the splinting period

- To optimise healing outcomes for the pulp and/or the periodontal ligament

- To improve function and provide comfort.

The International Association for Dental Traumatology (IADT) and the British Society of Paediatric Dentistry (BSPD) have provided guidelines for managing dental trauma injuries such as root fractures, alveolar fractures, subluxation injuries, extrusive luxation, lateral luxation, intrusive luxation and avulsion. The Dental Trauma guide, a website developed by the Copenhagen University and the IADT also contains treatment guidelines for managing trauma in primary and permanent teeth.

\section{Types of splints}

There are many different types of splints some of which are listed below:
- Composite and wire splints - the wire is secured to teeth with composite resin

- Composite and fishing line splints - this is where a fishing line replaces the wire

- Orthodontic wire and bracket splints orthodontic brackets are bonded to teeth with an orthodontic cement and connected with an orthodontic wire

- Fibre splints - usually a polyethylene or Kevlar fibre mesh bonded to teeth with an unfilled or composite resin

- Suture splints - suturing is required where there are multiple missing teeth or where conventional splinting is not possible

- Titanium trauma splint - the flexible titanium trauma splint has a rhomboid mesh structure and is just $0.2 \mathrm{~mm}$ thick. It is secured to teeth with composite resin and provides superior qualities when used for splinting traumatised teeth ${ }^{1}$

- Arch bar splints - these consist of metal bars bent into the shape of the arch and fixed in place with ligature wires

- Wire ligature splints - wire is tied around contact points and interproximal areas to stabilise traumatised teeth

- Composite splints - resin composite is directly applied to the surfaces of teeth as rigid splints. This type of splint is not recommended ${ }^{2}$

- Vacuum formed removable splints requires impression taking and preparation of models. An acrylic sheet is heated and drawn over the cast model under vacuum pressure to form the splint which can be trimmed to fit.

Composite and wire splints are the most commonly used in clinical practice.

The rigidity of the wire fixation is an important factor as it determines whether

\section{Table 1 Benefits of flexible splinting}

\section{Rigid Splinting}

Injured teeth are immobilised

Higher incidence of ankylosis

Poorer healing outcomes

Increased incidence of external root resorption

Increased incidence of pulp necrosis

\section{Flexible Splinting}

Provides some functional movement of traumatised teeth

Lower incidence of ankylosis

Better healing outcomes

Reduced incidence of external root resorption

Reduced incidence of pulp necrosis physiological movement of the teeth will be possible. Physiological movement of cells during the healing process enables maintenance of the viability of the periodontal ligament fibres.

Studies have indicated that for flexible composite and wire splints, the wire should have a diameter of no greater than $0.4 \mathrm{~mm} .^{1,2}$

Wires up to 0.016 (inches) i.e. $0.4 \mathrm{~mm}$ diameter are significantly more flexible than direct composite (rigid) splints and are thus better suited for the splinting traumatised teeth. ${ }^{3}$

Research also suggest that a diameter of 0.016 inches may be considered as a clinical threshold between flexible and non-flexible splints. ${ }^{3}$ The benefits can be found in Table 1.

Examples of flexible wires commonly used for splinting are; 0.016 inch diameter $\mathrm{Ni}-\mathrm{Ti}$ orthodontic archwire and 0.016 inch diameter stainless steel wire. The flexible titanium splint is also becoming more widely used although it has a relatively higher cost.

Benefits of using Titanium Trauma Splints

- Quicker - can be applied in much less time than a custom wire and composite splint

- Cleaner - removal and clean-up are much easier. The splint also facilities good oral hygiene

- More adaptable - the adaptability of the splint to adjacent teeth facilitates the etch-bond stage, as the splint can be easily tacked to adjacent teeth before application of flowable composite

- Better aesthetics and comfort - more comfortable than wire and composite resin splints

- Easier to use when working with paediatric patients - better co-operation as it is easier to use. This may mean avoiding a general anaesthetic session

- More effective - The rhomboid mesh structure helps to limit tooth mobility.

\section{References}

1. Aly A, Kenny D J. Titanium Trauma Splint: An Alternative Splinting Product. J Can Dent Assoc 2006; 72 :721-723.

2. Kahler B, Hu J-Y, Marriott-Smith CS. Splinting of teeth following trauma: a review and a new splinting recommendation. Aust Dent J 2016; 61: 59-73.

3. Kwan S C, Johnson J D, Cohenca N. The effect of splint material and thickness on tooth mobility after extraction and replantation using a human cadaveric model. Dent Traumol 2012; 28: 277-281.

Shola Todd 